\title{
A High Incidence of Metabolic Syndrome Traits in Mexicans Points at Obesity-Related Metabolic Dysfunction
}

This article was published in the following Dove Press journal: Diabetes, Metabolic Syndrome and Obesity: Targets and Therapy

\section{Omar Yaxmehen \\ Bello-Chavolla $\mathbb{D}^{1,2}$ \\ Arsenio Vargas-Vázquez $\mathbb{D}^{1,3, *}$ \\ Neftali Eduardo \\ Antonio-Villa $\mathbb{D}^{1,3, *}$ \\ Fabiola Mabel Del \\ Razo-Olvera (iD) \\ Daniel Elías-López' \\ Carlos A Aguilar-Salinas (1) 1,4,5 \\ For the Metabolic Syndrome \\ Study Group

\begin{abstract}
'Unidad de Investigación de Enfermedades Metabólicas, Instituto Nacional de Ciencias Médicas y Nutrición Salvador Zubirán, Mexico City, Mexico; ${ }^{2}$ Division of Research, Instituto Nacional de Geriatría, Mexico City, Mexico; ${ }^{3} \mathrm{MD} / \mathrm{PhD}$ (PECEM) Program, Facultad de Medicina, UNAM, Mexico City, Mexico; ${ }^{4}$ Division of Nutrition, Instituto Nacional de Ciencias Médicas y Nutrición Salvador Zubirán, Mexico City, Mexico; ${ }^{5}$ Tecnologico de Monterrey, Escuela de Medicina y Ciencias de la Salud, Mexico City, Mexico
\end{abstract}

*These authors contributed equally to this work

Correspondence: Carlos A Aguilar-Salinas Unidad de Investigación de Enfermedades Metabólicas, Instituto Nacional de Ciencias Médicas y Nutrición Salvador Zubirán, Vasco de Quiroga 15, Tlalpan, Distrito Federal, CP 14080, México Tel +525554870900, 5703

Email caguilarsalinas@yahoo.com
Introduction: Metabolic Syndrome (MS) is a construct relating to a series of metabolic dysfunctions attributable to insulin resistance and obesity. Here, we estimate the incidence of MS according to their individual components using a Mexican open-population cohort.

Methods: We evaluated data of 6144 Mexicans amongst whom 3340 did not have MS either by IDF or ATP-III definitions using data from an open-population cohort. We estimated the incidence of MS and each of its traits after a median follow-up of 2.24 (IQR 2.05-2.58) years and evaluated risk factors for MS incidence and each of its traits. We also explored individuals without any MS trait to evaluate trait and MS incidence after follow-up.

Results: We observed a high incidence of MS-IDF ( 115.11 cases per 1000 person-years, $95 \%$ CI 107.76-122.47), followed by MS-ATP-III (75.77 cases per 1000 person-years, 95\% CI). The MS traits with the highest incidence were low HDL-C and abdominal obesity, which was consistent for subjects without MS and those without any MS trait. When assessing predictors of MS incidence, obesity, insulin resistance, and increased apolipoprotein B levels predicted MS incidence. Weight loss $>5 \%$ of body weight and physical activity were the main protective factors. Obesity was a main determinant for incident MS traits in our population, with weight loss being also a protective factor for most MS traits.

Conclusion: We observed a high incidence of MS in apparently healthy Mexican adults. Low HDL-C and abdominal obesity were the most frequent incident MS traits, with obesity being the main determinant of its incidence.

Keywords: metabolic syndrome, obesity, incidence, Mexicans, metabolic risk

\section{Introduction}

The Metabolic Syndrome (MS) is a construct which relates to a series of metabolic dysfunctions attributable to the interplay between ectopic lipid accumulation, insulin resistance (IR), and obesity. ${ }^{1-3}$ It has been estimated that the prevalence of MS has been increasing steadily, posing an increased burden to healthcare systems, particularly in Mexico. ${ }^{4}$ Although IR has been proposed to be the main underlying factor, adipose tissue dysfunction has been shown to play an essential role in developing MS traits. ${ }^{5}$ MS is a useful tool to predict type 2 diabetes, arterial hypertension, non-alcoholic fatty liver disease, and cardiovascular disease. ${ }^{6,7}$ Reports of the estimated prevalence of MS and its traits in Mexican population have been consistently higher compared to European populations. ${ }^{8}$ Estimates of MS prevalence have been consistently increasing in the region, which has mostly been attributable to a series of multifactorial components in Mexicans, including genetic 
factors, an increase in the consumption of high carbohydrate diet, high prevalence of sedentary lifestyle, and insufficient public policies targeted to decrease the impact of MS in the general population. Therefore, MS will remain as a health problem for the foreseeable future., ${ }^{9,10}$

Despite its relevance, there are currently limited data on the incidence of MS and its traits in Mexicans, with most information being reported for isolated traits or relying only on prevalence data. The information generated in longitudinal follow-up cohorts would allow a better understanding of risk factors associated with the development of MS and its components in Mexicans, thus allowing for better monitoring of the trends of metabolic health in the general population. This information is critical to design public policies for the prevention of MS onset and its associated traits alongside with targeted strategies. In this work, we estimate the incidence of MS according to Adult Treatment Panel III (ATP-III) and International Diabetes Federation (IDF) definitions, alongside with the estimation of their individual components using a Mexican openpopulation cohort. Furthermore, we estimate the associated factors to predict MS and its individual components.

\section{Methods}

\section{Study Population}

The MS cohort was developed to estimate the risk for developing MS components in apparently healthy subjects in an urban population living in nine different cities in Mexico during a three-year period. ${ }^{11} \mathrm{We}$ included individuals aged 25-69 years, without type 2 diabetes, arterial hypertension, or other significant cardiovascular comorbidities. Individuals with diagnosed T2D or fasting plasma glucose $\geq 126 \mathrm{mg} / \mathrm{dl}$, coronary artery disease, cerebral vascular disease, alcoholism, taking corticosteroids, with liver disease, kidney dysfunction, or any life-threatening diseases that would prevent the two-year follow-up were excluded. Participants were interviewed to obtain medical history, sociodemographic information, dietary and physical activity habits, and anthropometric measurements, including weight, height, and waist circumference (WC). BP measurement was also performed using a manual sphygmomanometer after the participants remained seated for at least 5 minutes and refrained from consuming caffeine before the measurements. We obtained a $20 \mathrm{~mL}$ blood sample after 9-12 hour fast to measure of glucose, insulin, total and HDL cholesterol, triglyceride, apolipoprotein $\mathrm{B}$ (ApoB), and C-reactive protein (CRP). The short version of the International physical activity questionnaire (IPAQ) was used to measure physical activity; based on the reported time spent in moderate (including walking) and vigorous physical activity weighted by two, participants were categorized as being active $(\geq 150 \mathrm{~min} / \mathrm{wk})$ or inactive $(<150 \mathrm{~min} / \mathrm{wk}$ ) - according to WHO physical activity guidelines. These same evaluations were carried out after a minimum of two years follow-up. We recruited 7636 participants at baseline, of whom a total of 6144 participants agreed to continue with a follow-up visit and 22 participants died. Time to follow-up was estimated from time of recruitment up to the last follow-up or type 2 diabetes diagnosis, whichever occurred first.

\section{Ethics Statement}

The Research Ethics Committee and the Institutional Review Board of the Instituto Nacional de Ciencias Médicas y Nutrición Salvador Zubirán approved all study proceedings, with reference number Ref. 00778. All participants were provided with written informed consent and all proceedings were carried out in accordance with the Declaration of Helsinki.

\section{Biochemical and Anthropometric Evaluations}

Plasma glucose concentration was measured by an automated glucose analyzer (Yellow Springs Instruments Co.), serum insulin concentration was measured by using a chemiluminescent immunoassay (Beckman Coulter Access 2). Lipid concentrations (cholesterol, triglycerides, and HDL cholesterol), and ApoB, were measured using colorimetric assays (Unicel DxC 600 Synchron Clinical System Beckman Coulter). LDL-cholesterol was calculated using Martin's equation. HOMA2-IR was calculated using fasting glucose and insulin using the HOMA2 calculator released by the Diabetes Trials Unit, University of Oxford: HOMA Calculator; IR was defined as a HOMAIR value $>2.5$, as previously shown for the MS cohort. ${ }^{11}$ ApoB $>90$ th percentile was evaluated as a risk factor according to previously validated cut-off value in Mexican population. ${ }^{12}$

\section{MS Traits Definitions}

We defined MS according to IDF and ATP-III criteria. Individual MS components include the presence of central obesity (waist circumference $>102 \mathrm{~cm}$ in men and $>88 \mathrm{~cm}$ in women for ATP-III-MS criteria; $>90 \mathrm{~cm}$ in men and 
$>80 \mathrm{~cm}$ in women for IDF-MS criteria), hyperglycemia (fasting plasma glucose $\geq 100 \mathrm{mg} / \mathrm{dl}[5.6 \mathrm{mmol} / \mathrm{L}]$ ), low HDL-C (HDL-C $\leq 40 \mathrm{mg} / \mathrm{dl}[1.03 \mathrm{mmol} / \mathrm{L}]$ in men and $\leq 50 \mathrm{mg} / \mathrm{dl}$ [1.29 mmol/L] in women), hypertriglyceridemia (fasting plasma triglycerides $\geq 150 \mathrm{mg} / \mathrm{dl}[1.7 \mathrm{mmol} / \mathrm{L}]$ ) and arterial hypertension (peripheral arterial blood pressure $\geq 140 / \geq 90 \mathrm{mmHg}$ ). MS considered according to the IDF criteria was defined as the presence of central obesity plus two other components. ${ }^{13}$ MS considered according to the ATP-III criteria was defined as the presence of three or more components. ${ }^{14}$

Finally, incident type 2 diabetes was defined as medical diagnosis of type 2 diabetes, taking hypoglycemic medication and/or fasting glucose levels $\geq 7.0 \mathrm{mmol} / \mathrm{dl}(\geq 126 \mathrm{mg}$ / dl) according to American Diabetes Association guidelines. ${ }^{15}$ Weight changes were assessed during followup and calculated as percentage change compared to baseline weight; stable weight was defined as changes within $\pm 5 \%$ of baseline body weight whilst weight gain or loss was defined as an increase of a decrease higher than $5 \%$ of baseline body weight, respectively.

\section{Statistical Analysis}

\section{Metabolic Syndrome Incidence}

We estimated the incidence of MS using two approaches. First, we excluded all subjects who had at least one MS trait by either ATP-III or IDF to estimate the incidence of MS traits and MS in individuals without any metabolic alteration. Second, we excluded subjects who fulfilled either MS-ATP-III or MS-IDF to estimate the incidence of each MS definition after follow-up. We estimated incidence rates by dividing the number of new cases and standardized them per 1000 person-years of follow-up.

\section{Predictors of Incident MS and MS Traits}

To assess predictors at baseline of incident MS by ATP-III of IDF we fitted Cox proportional risk regression analyses considering incident MS or censoring in years, whichever occurred first in subjects without the respective definition at baseline; all models were adjusted for age and sex. Model assumptions were verified using Schoenfeld's residuals and items which did not fulfill the proportional risk assumption were either excluded from the model or included as a stratification parameter. Model and variable selection was conducted by minimization of the Bayesian Information Criterion (BIC), including metabolic variables standardized with mean 0 and standard deviation 1 or using previously validated categorizations. For models which predicted the incidence of MS traits in subjects without any definition of MS, we excluded subjects with the trait at baseline and performed an additional analysis in subjects without any MS-IDF criteria and BMI $<30 \mathrm{~kg} / \mathrm{m}^{2}$. A p-value $<0.05$ was considered as statistical significance threshold. All analyses were performed using R software version 3.6.2.

\section{Results}

\section{Subjects without Any MS-IDF Components}

Amongst 6144 subjects who completed follow-up (median of 2.24 years, IQR $2.05-2.54$ ), only 353 subjects (5.75\%) had no MS components according to MS-IDF definitions. The final analytical sample was comprised of 3340 adults without MS at baseline (Table 1). After 852.09 personyears, we identified 20 cases of incident MS-IDF (IR 23.47 per 1000 person-years, $95 \%$ CI $13.18-33.76$ ). The MS trait with the highest incidence was abdominal obesity, followed by low HDL-C, hypertriglyceridemia, arterial hypertension, and dysglycemia. Only 123 subjects did not develop any MS trait (33.99\%), 128 developed at least one (36.26\%), 81 at least two (22.95), 23 developed $3(6.5 \%)$, and only one subject developed all five traits. Notably, we observed that IDF incidence was higher in subjects aged 35-50 years and males in metabolically healthy subjects but higher in subjects $>50$ years and males for cases without MS (Supplementary Figures 1 and 2). The main predictors of incident IDF stratified by sex and adjusted by age were increasing BMI and HOMA2-IR (Table 1); using phenotypes the main predictors were elevated $\beta$-cell function (HOMA2- $\beta>100 \%$, HR $3.11,95 \%$ CI $1.24-7.79$ ) and obesity (HR $22.23,95 \% \mathrm{CI}$ $4.50-109.86$, c-statistic $=0.722$ ). When excluding cases without obesity, we identified only HOMA2-IR as a predictor of incident MS-IDF (Table 2).

\section{Subjects without Any MS-ATP-III Components}

A total of 698 subjects had no MS components according to MS criteria (11.36\%, Figure 1). After 1692.67 personyears of follow-up, we observed 42 cases of incident MSATP-III (IR 24.82, 95\% CI 17.32, 32.34). Low HDL-C was the most frequent incident MS trait, followed by hypertriglyceridemia, abdominal obesity by ATP-III, hypertension, and dysglycemia. After follow-up, 265 subjects remained free of ATP-III components (38.0\%), which 
Table I Comparison of Subjects at Baseline and at Follow-Up in Relation to the Presence or Absence of Metabolic Syndrome (MS) Definitions at Baseline or the Incidence of Said Traits in the MS Cohort in Subjects without MS by Either Definition at Baseline $(n=3340)$ and Analyzing Those without MS-IDF Criteria $(n=353)$ and MS-ATP-III Criteria $(n=698)$

\begin{tabular}{|c|c|c|c|c|c|c|c|}
\hline \multirow[t]{2}{*}{ Parameter } & \multicolumn{3}{|c|}{ No MS at Baseline } & \multicolumn{2}{|c|}{ No MS-IDF Components } & \multicolumn{2}{|c|}{ No-ATP-III Components } \\
\hline & $\begin{array}{c}\text { Baseline } \\
\text { Overall } \\
(n=3340)\end{array}$ & $\begin{array}{c}\text { Follow-Up No } \\
\text { MS (n=2345) }\end{array}$ & $\begin{array}{l}\text { MS by Either } \\
\text { Definition at } \\
\text { Follow-Up } \\
(n=995)\end{array}$ & $\begin{array}{c}\text { No-MS IDF } \\
\text { Components } \\
(n=353)\end{array}$ & $\begin{array}{l}\text { MS-IDF } \\
\text { Follow-Up } \\
(n=131)\end{array}$ & $\begin{array}{c}\text { No MS ATP- } \\
\text { III } \\
\text { Components } \\
(n=698)\end{array}$ & $\begin{array}{l}\text { MS-ATP-III } \\
\text { Follow-Up } \\
(n=42)\end{array}$ \\
\hline Age (years) & $40(34-47)$ & $39(33-46)$ & $41(35-48)$ & $38(32-44)$ & $39(35-48)$ & $39(33-45)$ & $38(34-49)$ \\
\hline Female sex (\%) & $2271(67.9)$ & $1646(70.19)$ & $625(62.8)$ & $230(65.2)$ & $92(70)$ & $464(66.5)$ & $29(69)$ \\
\hline Glucose (mg/dl) & $83(77-89)$ & $83(76-89)$ & $86(77-95)$ & $81(74-86)$ & $83(74-92)$ & 81 (75-87) & $92(80-102)$ \\
\hline Triglycerides (mg/dl) & $12 \mid(91-164)$ & $114(86-148)$ & $168(128-227)$ & $98(76-121)$ & $148(102-184)$ & $99(78-122)$ & $157(109-191)$ \\
\hline HDL-C (mg/dl) & $49.2(11.9)$ & $50.46(12.29)$ & $38.6(10.4)$ & $57($ (II.2) & $42.5(8.6)$ & $57(10.1)$ & $42(7.9)$ \\
\hline $\mathrm{SBP}(\mathrm{mmHg})$ & $114(110-119)$ & 114 (110-119) & $120(110-130)$ & 114 (110-118) & $112(110-130)$ & 114 (110-119) & $118(110-129)$ \\
\hline $\mathrm{DBP}(\mathrm{mmHg})$ & $75(70-80)$ & $75(70-80)$ & $80(70-86)$ & $75(70-78)$ & $80(70-90)$ & $76(70-79)$ & $80(70-90)$ \\
\hline Waist Circunference (cm) & $89(82-95)$ & $88(81-94)$ & $94(88-101)$ & $79(75-86)$ & 91 (86-97) & $84(79-88)$ & $94(88-103)$ \\
\hline BMI (kg/m2) & $27.5(4.2)$ & $26.94(3.91)$ & $29.2(4.5)$ & $24.5(2.4)$ & $28.9(4.0)$ & $25.5(2.6)$ & $28.1(3.3)$ \\
\hline Waist-to-height ratio & $0.55(0.52-0.59)$ & $0.54(0.5 \mathrm{I}-0.59)$ & $0.58(0.55-0.62)$ & $0.49(0.47-0.52)$ & $0.58(0.55-0.61)$ & $0.53(0.49-0.55)$ & $0.59(0.56-0.62)$ \\
\hline Total colesterol (mg/dl) & $200(174-229)$ & $197(172-225)$ & $196(|69-22|)$ & $198(|77-22|)$ & $195(170-219)$ & $200(178-222)$ & $197(174-220)$ \\
\hline LDL-C (mg/dl) & $125(30.4)$ & $123.67(30.80)$ & $123(31.7)$ & $122(31.1)$ & $122.3(30.9)$ & $125(31.1)$ & $123(30.9)$ \\
\hline Apolipoprotein-B (mg/dl) & $102.8(26.4)$ & $99.24(25.30)$ & $106(26.5)$ & $92.7(22.1)$ & $100(23.6)$ & $94(22.5)$ & $102(23.4)$ \\
\hline $\begin{array}{l}\text { Apolipoprotein } B \geq 90^{\text {th }} \\
\text { percentile (\%) }\end{array}$ & |49| (44.7) & $900(38.46)$ & $514(52.12)$ & $103(29.8)$ & $57(44.2)$ & $211(30.2)$ & $21(5 \mid .2)$ \\
\hline C-Reactive Protein (mg/dl) & $2.53(2.48)$ & $2.42(2.44)$ & $3.16(2.87)$ & $1.55(1.78)$ & $2.94(2.3)$ & $1.89(2.1)$ & $3.05(1.4 I)$ \\
\hline HOMA2-IR & $1.73(1.18-2.52)$ & $1.64(1.12-2.33)$ & $1.3(0.8-1.9)$ & $1.22(0.88-1.70)$ & $1.20(0.75-1.50)$ & $1.32(0.93-1.90)$ & $1.2(0.9-1.6)$ \\
\hline Weight change (\%) & - & $\begin{array}{c}0.14(-3.22 \\
3.82)\end{array}$ & $1.46(-1.4 \mathrm{I}, 4.63)$ & - & $\begin{array}{c}1.67(-0.35 \\
5.20)\end{array}$ & - & $1.77(0.0,5.76)$ \\
\hline Weight gain >5\% (\%) & - & $402(19.03)$ & $224(22.88)$ & - & $36(27.91)$ & - & $14(33.33)$ \\
\hline Weight loss >5\% (\%) & - & $330(15.63)$ & $80(8.17)$ & - & $10(7.75)$ & - & $2(4.76)$ \\
\hline
\end{tabular}

was reduced to 161 if we considered IDF criteria for abdominal obesity (23.1\%); 264 subjects developed at least one MS trait (38.8\%), 127 two traits (18.2\%), 37 developed three $(5.3 \%)$, and 5 developed 4 traits $(0.7 \%)$. We observed increasing MS-ATP-III incidence by increasing age categories and in females compared to males (Supplementary Figures 1 and 2). For incident MSATP-III in subjects without MS components we identified as predictors stratified by sex increasing ApoB and BMI (Table 2); when using phenotypes, a higher risk was

Table 2 Incidence of Metabolic Syndrome, Metabolic Syndrome Components and Type 2 Diabetes Comparing Individuals without Any MS Criteria, Individuals without MS-IDF Criteria and Individuals without MS-ATP-III Criteria After Follow-Up in Apparently Healthy Mexican Adults

\begin{tabular}{|c|c|c|c|c|c|c|}
\hline \multirow[t]{2}{*}{ Status } & \multicolumn{2}{|c|}{ No MS IDF Components (N=353) } & \multicolumn{2}{|c|}{ No MS ATP-III Components $(\mathrm{N}=698)$} & \multicolumn{2}{|c|}{$\begin{array}{c}\text { No-MS by Either Definition at } \\
\text { Baseline }(\mathrm{N}=3340)\end{array}$} \\
\hline & $\begin{array}{l}\text { Accumulated } \\
\text { Incidence (\%) }\end{array}$ & $\begin{array}{l}\text { Incidence Rate } \\
\qquad(95 \% \mathrm{CI})\end{array}$ & $\begin{array}{l}\text { Accumulated } \\
\text { Incidence (\%) }\end{array}$ & $\begin{array}{l}\text { Incidence Rate } \\
\qquad(95 \% \mathrm{CI})\end{array}$ & $\begin{array}{l}\text { Accumulated } \\
\text { Incidence (\%) }\end{array}$ & $\begin{array}{l}\text { Incidence Rate } \\
\qquad(95 \% \mathrm{CI})\end{array}$ \\
\hline Incident MS-IDF & $20(5.3)$ & $23.47(13.18-33.76)$ & $82(11.7)$ & $48.47(37.98-58.96)$ & $94 I / 3340(28.1)$ & II5.II (107.76-122.47) \\
\hline Incident MS-ATP-III & $32(9.1)$ & $37.55(24.54-50.57)$ & $42(6.0)$ & $24.82(17.32-32.34)$ & $619 / 3340(18.5)$ & $75.77(69.76-81.69)$ \\
\hline Incident hypertension & $32(9.1)$ & $37.55(24.54-50.57)$ & $82(11.7)$ & $48.47(37.98-58.96)$ & $435 / 2999(14.5)$ & 59.31 (53.73-64.88) \\
\hline Incident dysglycemia & $14(3.4)$ & $16.43(7.82-25.04)$ & $38(5.4)$ & $22.46(15.32-29.6 I)$ & $243 / 3263(7.4)$ & $30.70(26.89-34.5 \mathrm{I})$ \\
\hline Incident diabetes & $4(1.1)$ & $4.69(0.09-9.29)$ & $12(1.7)$ & $7.09(3.08-11.11)$ & $87 / 3340(2.6)$ & $10.32(8.15-12.49)$ \\
\hline Incident hypertriglyceridemia & $84(23.8)$ & $98.58(77.50-119.66)$ & $165(24.4)$ & $97.54(82.65-112.42)$ & $595 / 2239(26.6)$ & $109.09(100.33-117.86)$ \\
\hline Incident low HDL-C & $110(31.2)$ & $129.09(104.97-153.22)$ & $250(35.8)$ & $147.78(129.46-166.10)$ & $869 / 2079(41.8)$ & $170.60(159.26-181.94)$ \\
\hline Incident abdominal obesity IDF & $124(35.1)$ & 145.53 (119.92-171.14) & $390(55.9)$ & $230.54(207.66-253.42)$ & $5 I 5 / I 24 I(4 I .5)$ & $169.21(154.60-183.83)$ \\
\hline Incident abdominal obesity ATP-3 & $29(8.2)$ & $34.03(21.65-46.42)$ & $114(16.3)$ & $67.39(55.02-79.76)$ & $391 / 2324(16.8)$ & $69.10(62.25-75.95)$ \\
\hline
\end{tabular}




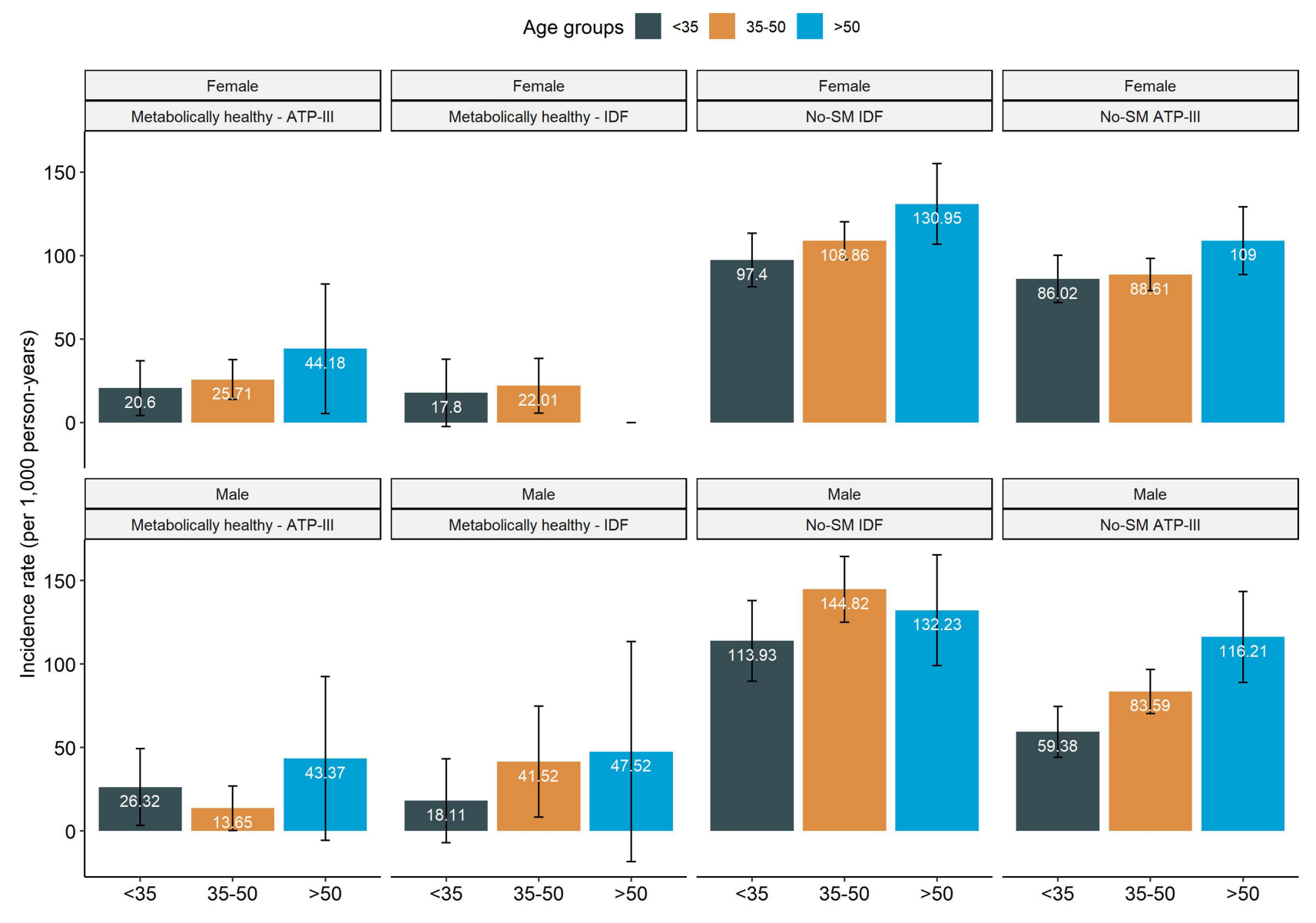

Figure I Incidence rates of metabolic syndrome (MS) per 1000 person-years using IDF and ATP-III definitions in individuals without MS components, and those without any MS definition stratified by age and sex.

attributable to $\mathrm{ApoB}>90^{\text {th }}$ percentile (HR 3.19, 95\% CI 1.69-6.02), overweight (HR 3.23, 95\% CI 1.45-7.19) and obese subjects (HR 10.21, 95\% CI 3.25-32.03, c-statistic $=0.747$ ).

\section{Subjects without MS by Any Definition}

At baseline 2038 (33.2\%) subjects had MS-ATP-III and 2699 (43.9\%) had MS-IDF; a total of 3340 subjects did not meet MS criteria by either definition. Amongst those without MS by any definition 77 subjects had dysglycemia (2.3\%), 2099 had abdominal obesity by MS-IDF (62.8\%) and 1016 by MS-ATP-III (30.4\%), 341 had hypertension (10.2\%), 1261 had low HDL-C (37.8\%), and 1101 had hypertriglyceridemia $(33.0 \%)$. Using MS-IDF criteria 1095 had two MS components (37.8\%) and 1892 had one (56.6\%); conversely, using ATP-III criteria 1154 had two MS components (34.56\%) and 1488 (44.55\%) had one. The most frequent incident MS trait was MS-IDF defined abdominal obesity, followed by low HDL-C, hypertriglyceridemia, MS-ATP-III abdominal obesity, arterial hypertension, and dysglycemia (Table 2). Some metabolic phenotypes showed an increased risk of incident MS-IDF. As expected, having one MS criteria or two MS criteria had a greater risk compared to none. Weight gain $>5 \%$, higher WHtR, BMI, HOMA2-IR, and ApoB were associated with an increased risk, whilst weight loss $>5 \%$ and physical activity were protective factors. For MS-ATP-III, predictors were having one or two MS components, BMI, HOMA2IR, physical activity, older age, and female sex (Table 3).

\section{Predictors of Incident Individual MS Traits}

Table 2 shows the incidence of each one of the metabolic syndrome traits and its different definitions; population was stratified according to their number of metabolic abnormalities at the initial visit. The highest incidence for individual MS traits was found for abdominal obesity, followed by low HDL-C. Next, we investigated factors related to the incidence of each MS trait in subjects who did not fulfill any MS definition (Figure 2). For incident hyperglycemia, the main predictors were obesity, 
Table 3 Cox Proportional Hazard Regression Models Assessing Risk Factors for of Incident MS in Individuals without Any MS Criteria (No-MS) and Incident IDF and ATP-3 in Individuals

\begin{tabular}{|c|c|c|c|c|c|c|}
\hline Model Parameters & Variable & $\begin{array}{c}\beta- \\
\text { Coefficient }\end{array}$ & Wald & HR & $95 \% \mathrm{Cl}$ & p-value \\
\hline \multirow{2}{*}{ No MS criteria - IDF c-statistic $=0.748$} & HOMA2-IR & 0.551 & 2.690 & 1.735 & $1.16 \mathrm{I}-2.592$ & 0.007 \\
\hline & $\mathrm{BMI}>\mathrm{ISD}$ & 0.236 & 2.604 & 1.266 & $1.060-1.512$ & 0.009 \\
\hline $\begin{array}{l}\text { No MS criteria }- \text { IDF and BMI }<30 \mathrm{~kg} / \mathrm{m}^{2} \\
\text { c-statistic }=0.678\end{array}$ & HOMA2-IR & 0.614 & 2.961 & 1.848 & $1.23 \mathrm{I}-2.776$ & 0.003 \\
\hline \multirow[t]{2}{*}{ No MS criteria - ATP-3 c-statistic $=0.760$} & $\mathrm{BMI}>\mathrm{ISD}$ & 0.223 & 3.001 & 1.250 & $1.126-1.387$ & $<0.001$ \\
\hline & Apolipoprotein B >ISD & 0.019 & 4.194 & 1.019 & $1.006-1.031$ & $<0.001$ \\
\hline \multirow[t]{8}{*}{ Incident MS ATP-3 c-statistic $=0.700$} & No MS components & \multicolumn{5}{|c|}{ Reference } \\
\hline & I MS component & 0.733 & 4.318 & 2.081 & $1.492-2.901$ & $<0.001$ \\
\hline & 2 MS components & 1.156 & 6.749 & 3.178 & $2.272-4.446$ & $<0.001$ \\
\hline & $\mathrm{BMI}>\mathrm{ISD}$ & 0.217 & 6.622 & 1.243 & $1.165-1.325$ & $<0.001$ \\
\hline & HOMA2-IR & 0.101 & 2.108 & 1.107 & $1.007-1.216$ & 0.035 \\
\hline & Apolipoprotein B >ISD & 0.175 & 4.369 & 1.191 & $1.101-1.289$ & $<0.001$ \\
\hline & Physical activity & -0.238 & -2.829 & 0.788 & $0.668-0.930$ & 0.005 \\
\hline & Male sex & -0.285 & -3.002 & 0.752 & $0.625-0.906$ & 0.004 \\
\hline \multirow[t]{9}{*}{ Incident IDF c-statistic $=0.656$} & No SM components & \multicolumn{5}{|c|}{ Reference } \\
\hline & I SM component & 0.648 & 6.603 & 1.913 & $1.578-2.319$ & $<0.001$ \\
\hline & 2 SM components & 0.753 & 5.729 & 2.123 & $\mathrm{I} .64 \mathrm{I}-2.746$ & $<0.001$ \\
\hline & HOMA2-IR & 0.106 & 2.501 & 1.111 & $1.023-1.207$ & 0.012 \\
\hline & $\mathrm{WHtR}>\mathrm{ISD}$ & 0.097 & 2.236 & 1.102 & $1.0170-1.199$ & 0.025 \\
\hline & Apolipoprotein B >I & 0.220 & 6.812 & 1.246 & $1.013-1.328$ & $<0.001$ \\
\hline & SD & & & & & \\
\hline & $\mathrm{BMI}>\mathrm{ISD}$ & 0.090 & 2.291 & 1.094 & $1.013-1.182$ & 0.022 \\
\hline & Physical activity & -0.140 & -2.042 & 0.869 & $0.760-0.994$ & $0.04 I$ \\
\hline
\end{tabular}

Abbreviations: HOMA2-IR, Homeostasis Model Assessment for Insulin Resistance; BMI, body mass index; MS, metabolic syndrome; IDF, International Diabetes Federation; ATP-III, Adult Treatment Panel III; SD, standard deviation from the mean; WHtR, waist-to-height ratio.

overweight, and age, whilst weight loss was a protective factor. Incident hypertriglyceridemia was predicted in subjects with $\mathrm{ApoB}>90^{\text {th }}$ percentile, whilst younger age $(<1$ SD from the mean) and weight loss $>5 \%$ were protective factors. Similarly, for arterial hypertension higher risk was observed in obesity, overweight, FPG, and age. Risk of developing low HDL-C was associated with ApoB $>90^{\text {th }}$ percentile, obesity, and overweight, whilst lower risk was attributable to weight loss and younger age. For abdominal obesity by either ATP-III or IDF criteria, higher risk was observed in overweight, obesity, visceral obesity (WHtR $>1 \mathrm{SD}$ ), and weight gain; protective factors included weight loss and physical activity primarily in individuals with visceral obesity, with these predictors having superior performance in ATP-III abdominal obesity (c-statistic $=0.641)$ compared to IDF (c-statistic $=0.608)$.

\section{Discussion}

Here, we present the largest report on the incidence of Metabolic Syndrome and its traits in Mexican adults. Our results point at obesity as a main driver for the incidence of Metabolic Syndrome and most of its traits as well as the relevance of intervention strategies related to weight control and physical activity in ameliorating metabolic burden in the region. Interestingly, we observed that in subjects without any MS traits, obesity, insulin resistance, and high levels of apolipoprotein B were still predictors of incident MS and its traits, indicating that these three metabolic abnormalities might be relevant predictors of metabolic dysfunction in otherwise metabolically healthy Mexican adults. Our findings advocate for more strict policies in ameliorating the impact of obesity to reduce metabolic dysfunction and the promotion of healthy lifestyles including physical activity and weight control as means to reduce the burden of metabolic diseases in Mexico. 

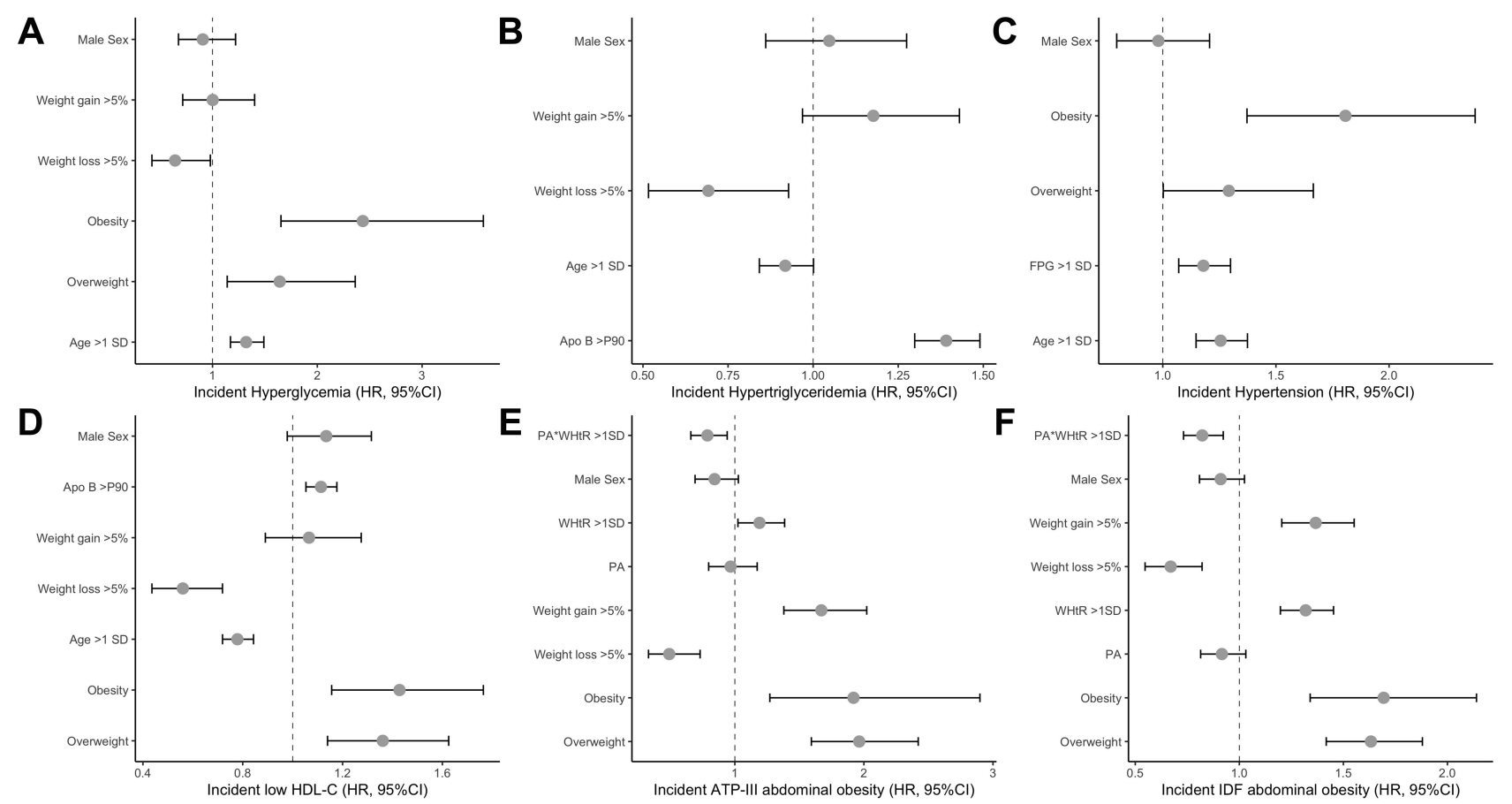

Figure 2 Predictors of incident metabolic syndrome (MS) traits in subjects without any definition of the metabolic syndrome using Cox Proportional Hazard Regression models. Subjects having the trait at baseline were excluded from each individual analysis. We show predictors for incident hyperglycemia (A), hypertriglyceridemia (B), hypertension (C), low HDL-C (D), ATP-III abdominal obesity (E) and IDF abdominal obesity (F).

Abbreviations: BMI, body mass index; FPG, fasting plasma glucose; FTG, fasting triglycerides; DBP; diastolic blood pressure, WHtR, waist-to-height ratio; PA, physical activity.

Few reports are available about the incidence of the metabolic syndrome and its individual traits (except for diabetes) worldwide. Most of the cohort studies have described the incidence of the components separately, without integrating them into any of the versions of the metabolic syndrome. In the ARIC study, it was observed that the incidence of the syndrome was determined by changes in body weight and by baseline body mass index. The 10-year risk for meeting the NCEP-ATP-III definition is 4.5 times greater in people with obesity compared to lean subjects. The incidence of the components varies according to BMI and ethnicity. No available information exists in Mexicans and almost all reports have been focused on prevalence but not on incidence. The most prevalent MS trait in Mexicans is abdominal obesity, with most subjects having at least one more associated MS trait; ${ }^{10}$ our data show that abdominal obesity is also the MS trait with the biggest incidence. Chronic exposure to a positive net caloric intake, increased genetic predisposition, and sedentary lifestyles are significant contributors to the appearance and progression of MS and each of its traits, as confirmed by our results. ${ }^{16}$ Notably, abdominal obesity has a significant contribution to the MS construct and previous research from our group demonstrated the role of including a more precise visceral fat estimation within the MS construct. ${ }^{17,18}$ Visceral adiposity has also been linked to metabolic dysfunction in metabolically healthy obesity, whereby a transition to metabolically unhealthy phenotypes is mostly linked to increased visceral fat accumulation and unhealthy expansion of adipose tissue during weight gain. ${ }^{19,20}$ Metabolic dysfunction linked to increased visceral fat accumulation has been associated with an overall pro-inflammatory process and adverse adipokine profiles associated with increased risk of metabolic and cardiovascular diseases. ${ }^{21}$ Similarly, obesity has been observed to be associated with impaired redox homeostasis and oxidative stress, which likely contribute to adipose tissue dysfunction and peripheral insulin resistance. ${ }^{22}$ Our finding of a beneficial effect of physical activity primarily in individuals with visceral obesity as assessed by increased WHtR confirmed previous findings of a particular beneficial effect of physical activity in reducing visceral adiposity. ${ }^{23}$

Low HDL-C was the MS trait with the second largest incidence. This observation is in accordance with the high prevalence of low HDL cholesterol levels in each one of the national health surveys done in Mexico. ${ }^{24}$ Its incidence is high in subjects with or without other metabolic 
syndrome traits, which goes along with a rather large incidence of hypertriglyceridemia. We observed that increased ApoB levels is a predictor of hypertriglyceridemia and low HDL-C incidence. Increased ApoB levels along with MS and IR have been previously linked to familial combined hyperlipidemia (FCHL), a primary dyslipidemia with high prevalence in Mexican population. ${ }^{25,26}$ Given that the MS cohort did not include extensive family studies of subjects with dyslipidemia, confirmation of the presence of FCHL at an individual level is not feasible; however, incident hypertriglyceridemia in subjects with increased ApoB and a previously normal lipid profile is a hallmark of this condition, particularly in younger subjects. ${ }^{27}$ A similar genetic effect could be attributable to the high incidence of abdominal obesity, which is particularly linked to insulin-resistant states. The SIGMA consortium reported a common risk variant in SLC16A11 which increases the risk of $\mathrm{T} 2 \mathrm{D}$ and has been associated with decreased insulin sensitivity and large-sized adipocytes, which could point at metabolic disturbances associated with adipose tissue dysfunction and peripheral insulin resistance. ${ }^{28,29}$ Notably, both diseases also increase the risk of non-alcoholic fatty liver disease (NAFLD), a trait which is also common in Mexican adults with comorbid MS and which increases cardio-metabolic risk. ${ }^{30,31}$ Prospective studies should focus on mechanistic approaches which investigate the risk associated with FCHL, T2D and genetic risk variants which increase cardio-metabolic risk and its relationship in the development of metabolically unhealthy states including IR, NAFLD, and adipose tissue dysfunction. These evaluations are still required, particularly in Mexican and Latin American populations, who might have higher risk compared to other populations.

Our study had some strengths and limitations. Ours is the largest open-population cohort investigating the incidence of MS and its traits in Mexicans and in Latin Americans, a population with intrinsically higher metabolic burden and a high incidence of cardio-metabolic events. In this light, a potential limitation of our approach is the fact that the MS cohort comprises higher risk profiles which might overestimate the incidence of MS and its traits but, on the other hand, achieves sufficient power to estimate potential predictors of disease onset. An additional consideration to contextualize the results is the lack of nationally representative data on MS incidence, which could be useful to inform specific regional needs and will remain an area of opportunity for future studies related to MS traits. More extensive assessments of body composition in relation to MS incidence are also required to investigate which phenotypes have higher likelihood of developing metabolic dysfunction and how changes in adipose tissue distribution modify these risk profiles. ${ }^{32}$

In conclusion, we observed a high incidence of MS and its traits in Mexican adults from an open population cohort. Predictors of incident MS in subjects with and without any MS criteria point at metabolic dysfunction related to whole-body fat distribution and obesity, in particular its interaction with lipoprotein metabolism and whole-body insulin sensitivity. Physical activity and weight loss are significant protective factors for most MS traits, and they may be particularly useful in subjects with visceral obesity in whom adipose tissue dysfunction might drive the appearance of metabolically unhealthy phenotypes. Given the increased cardio-metabolic burden observed in Mexicans, mechanistic studies which investigate MS incidence and its traits from a systems biology standpoint will be pivotal in unraveling pathophysiological mechanisms underlying these phenomena. Further studies should focus on evaluating the mechanistic role of adipose tissue function on the onset of metabolic health, with a focus on adipose tissue compared to peripheral insulin resistance, adipokine profiles and the interaction between adipose tissue phenotypes with ectopic fat accumulation and its role in worsening metabolic health.

\section{Metabolic Syndrome Study Group}

The metabolic syndrome study group is composed by: Olimpia Arellano-Campos, Donaji V. Gómez-Velasco, Omar Yaxmehen Bello-Chavolla, Ivette Cruz-Bautista, Marco A. Melgarejo-Hernandez, Paloma Almeda-Valdés, Alexandro J. Martagón, Liliana Muñoz-Hernandez, Luz E. Guillén, José de Jesús Garduño-García, Ulices Alvirde, Yukiko Ono-Yoshikawa, Ricardo Choza-Romero, Leobardo Sauque-Reyna, Ma. Eugenia Garay-Sevilla, Juan M. Malacara-Hernandez, María Teresa Tusié-Luna, Luis Miguel Gutierrez-Robledo, Francisco J Gómez-Pérez, Rosalba Rojas and Carlos A. Aguilar-Salinas.

\section{Data Sharing Statement}

Data is available from the corresponding author upon reasonable request.

\section{Acknowledgments}

All authors approved the submitted version. All the authors would like to thank the staff of the Endocrinology and 
Metabolism Department for all their support, particularly to Luz Elizabeth Guillen-Pineda, Maria Del Carmen MorenoVillatoro and Adriana Cruz-Lopez. We are thankful to the study volunteers for all their work and support throughout the realization of the study. AVV and NEAV are enrolled at the PECEM program of the Faculty of Medicine at UNAM and are supported by CONACyT.

\section{Author Contributions}

All authors made a significant contribution to the work reported, whether that is in the conception, study design, execution, acquisition of data, analysis and interpretation, or in all these areas; took part in drafting, revising or critically reviewing the article; gave final approval of the version to be published; have agreed on the journal to which the article has been submitted; and agree to be accountable for all aspects of the work.

\section{Disclosure}

The authors report no conflicts of interest in this work.

\section{References}

1. Barazzoni R, Gortan Cappellari G, Ragni M, Nisoli E. Insulin resistance in obesity: an overview of fundamental alterations. Eat Weight Disord. 2018;23:149-157. doi:10.1007/s40519-018-0481-6

2. Carr DB, Utzschneider KM, Hull RL, et al. Intra-abdominal fat is a major determinant of the National Cholesterol Education Program Adult Treatment Panel III criteria for the metabolic syndrome. Diabetes. 2004;53(8):2087-2094. doi:10.2337/diabetes.53.8.2087

3. Abel ED, O'Shea KM, Ramasamy R. Insulin resistance: metabolic mechanisms and consequences in the heart. Arterioscler Thromb Vasc Biol. 2012;32(9):2068-2076. doi:10.1161/ATVBAHA.111.241984

4. Gutiérrez-Solis AL, Datta Banik S, Méndez-González RM. Prevalence of metabolic syndrome in Mexico: a systematic review and meta-analysis. Metab Syndr Relat Disord. 2018;16(8):395-405. doi:10.1089/met.2017.0157

5. Shah RV, Murthy VL, Abbasi SA, et al. Visceral adiposity and the risk of metabolic syndrome across body mass index: the MESA Study. JACC Cardiovasc Imaging. 2014;7:1221-1235. doi:10.1016/ j.jemg.2014.07.017

6. Shin J-A, Lee J-H, Lim S-Y, et al. Metabolic syndrome as a predictor of type 2 diabetes, and its clinical interpretations and usefulness. J Diabetes Investig. 2013;4:334-343. doi:10.1111/jdi.12075

7. Ballantyne CM, Hoogeveen RC, McNeill AM, et al. Metabolic syndrome risk for cardiovascular disease and diabetes in the ARIC study. Int J Obes. 2008;32(Suppl 2):S21-S24. doi:10.1038/ijo.2008.31

8. Aguilar-Salinas CA, Rojas R, Gómez-Pérez FJ, et al. High prevalence of metabolic syndrome in Mexico. Arch Med Res. 2004;35:76-81. doi:10.1016/j.arcmed.2003.06.006

9. Barquera S, Campos-Nonato I, Aguilar-Salinas C, Lopez-Ridaura R, Arredondo A, Rivera-Dommarco J. Diabetes in Mexico: cost and management of diabetes and its complications and challenges for health policy. Global Health. 2013;9(1):3. doi:10.1186/1744-8603-9-3

10. Rojas-Martínez R, Aguilar-Salinas CA, Jiménez-Corona A, GómezPérez FJ, Barquera S, Lazcano-Ponce E. Prevalence of obesity and metabolic syndrome components in Mexican adults without type 2 diabetes or hypertension. Salud Publica Mex. 2012;54:7-12.
11. Arellano-Campos O, Gómez-Velasco DV, Bello-Chavolla OY, et al. Development and validation of a predictive model for incident type 2 diabetes in middle-aged Mexican adults: the metabolic syndrome cohort. BMC Endocr Disord. 2019;19(1):41. doi:10.1186/s12902-019-0361-8

12. Valles V, Aguilar-Salinas CA, Gómez-Pérez FJ, et al. Apolipoprotein $B$ and A-I distribution in Mexican urban adults: results of a nationwide survey. Metab Clin Exp. 2002;51(5):560-568. doi:10.1053/meta.2002.31977

13. Ford ES. Prevalence of the metabolic syndrome defined by the international diabetes federation among adults in the. US Diabetes Care. 2005;28(11):2745-2749. doi:10.2337/diacare.28.11.2745

14. Grundy SM, Cleeman JI, Daniels SR, et al. Diagnosis and management of the metabolic syndrome: an American heart association/national heart, lung, and blood institute scientific statement. Circulation. 2005;112 (17):2735-2752. doi:10.1161/CIRCULATIONAHA.105.169404

15. American Diabetes Association. 2. Classification and diagnosis of diabetes: standards of medical care in diabetes-2020. Diabetes Care. 2020;43:S14-S31. doi:10.2337/dc20-S002

16. Aguilar-Salinas CA, Viveros-Ruiz T. Recent advances in managing/ understanding the metabolic syndrome. [version 1; peer review: 3 approved]. F1000Res. 2019;8:8. doi:10.12688/f1000research.17122.1

17. Bello-Chavolla OY, Antonio-Villa NE, Vargas-Vázquez A, et al. Metabolic Score for Visceral Fat (METS-VF), a novel estimator of intra-abdominal fat content and cardio-metabolic health. Clin Nutr. 2020;39(5):1613-1621. doi:10.1016/j.clnu.2019.07.012

18. Antonio-Villa NE, Bello-Chavolla OY, Vargas-Vázquez A, Mehta R, Aguilar-Salinas CA; Metabolic Syndrome Study Group. The combination of insulin resistance and visceral adipose tissue estimation improves the performance of metabolic syndrome as a predictor of type 2 diabetes. Diabet Med. 2020;37(7):1192-1201. doi:10.1111/dme.14274

19. Vishvanath L, Gupta RK. Contribution of adipogenesis to healthy adipose tissue expansion in obesity. J Clin Invest. 2019;129 (10):4022-4031. doi:10.1172/JCI129191

20. Kang YM, Jung CH, Cho YK, et al. Visceral adiposity index predicts the conversion of metabolically healthy obesity to an unhealthy phenotype. PLoS One. 2017;12(6):e0179635. doi:10.1371/journal. pone. 0179635

21. Hajer GR, van Haeften TW, Visseren FLJ. Adipose tissue dysfunction in obesity, diabetes, and vascular diseases. Eur Heart J. 2008;29 (24):2959-2971. doi:10.1093/eurheartj/ehn387

22. Choromańska B, Myśliwiec P, Łuba M, et al. A longitudinal study of the antioxidant barrier and oxidative stress in morbidly obese patients after bariatric surgery. Does the metabolic syndrome affect the redox homeostasis of obese people? J Clin Med. 2020;9(4):9. doi:10.3390/jcm9040976

23. Cameron N, Godino J, Nichols JF, Wing D, Hill L, Patrick K. Associations between physical activity and BMI, body fatness, and visceral adiposity in overweight or obese Latino and non-Latino adults. Int J Obes. 2017;41(6):873-877. doi:10.1038/ijo.2017.49

24. Aguilar-Salinas CA, Olaiz G, Valles V, et al. High prevalence of low HDL cholesterol concentrations and mixed hyperlipidemia in a Mexican nationwide survey. J Lipid Res. 2001;42(8):1298-1307. doi:10.1016/S0022-2275(20)31581-9

25. Bello-Chavolla OY, Kuri-García A, Ríos-Ríos M, et al. Familial combined hyperlipidemia: current knowledge, perspectives, and controversies. Rev Invest Clin. 2018;70:224-236. doi:10.24875/ RIC. 18002575

26. Huertas-Vazquez A, Aguilar-Salinas C, Lusis AJ, et al. Familial combined hyperlipidemia in Mexicans: association with upstream transcription factor 1 and linkage on chromosome 16q24.1. Arterioscler Thromb Vasc Biol. 2005;25(9):1985-1991. doi:10.1161/ 01.ATV.0000175297.37214.a0

27. Pei W, Sun Y, Lu B, et al. Apolipoprotein B is associated with metabolic syndrome in Chinese families with familial combined hyperlipidemia, familial hypertriglyceridemia and familial hypercholesterolemia. Int $J$ Cardiol. 2007;116(2):194-200. doi:10.1016/j.ijcard.2006.03.045 
28. Almeda-Valdes P, Gómez Velasco DV, Arellano Campos O, et al. The SLC16A11 risk haplotype is associated with decreased insulin action, higher transaminases and large-size adipocytes. Eur J Endocrinol. 2019;180(2):99-107. doi:10.1530/EJE-18-0677

29. Williams AL, Jacobs SBR, Moreno-Macías H; SIGMA Type 2 Diabetes Consortium, et al. Sequence variants in SLC16A11 are a common risk factor for type 2 diabetes in Mexico. Nature. 2014;506:97-101. doi:10.1038/nature12828

30. Gill C, Vatcheva KP, Pan -J-J, et al. Frequency of nonalcoholic fatty liver disease and subclinical atherosclerosis among young Mexican americans. Am J Cardiol. 2017;119(11):1717-1722. doi:10.1016/j. amjcard.2017.03.010
31. Kallwitz ER, Tayo BO, Kuniholm MH, et al. American ancestry is a risk factor for suspected nonalcoholic fatty liver disease in hispanic/ latino adults. Clin Gastroenterol Hepatol. 2019;17(11):2301-2309. doi:10.1016/j.cgh.2019.02.007

32. Pi-Sunyer $X$. Changes in body composition and metabolic disease risk. Eur J Clin Nutr. 2019;73(2):231-235. doi:10.1038/s41430-0180320-x

\section{Publish your work in this journal}

Diabetes, Metabolic Syndrome and Obesity: Targets and Therapy is an international, peer-reviewed open-access journal committed to the rapid publication of the latest laboratory and clinical findings in the fields of diabetes, metabolic syndrome and obesity research. Original research, review, case reports, hypothesis formation, expert opinion and commentaries are all considered for publication. The manuscript management system is completely online and includes a very quick and fair peer-review system, which is all easy to use. Visit http://www.dovepress.com/testimonials.php to read real quotes from published authors. 